June 23, 1998

HKS-NT-FR-98/3-SE

CERN-TH/98-173

\title{
Unitarity of Strings and Non-compact Hermitian Symmetric Spaces
}

\author{
Stephen Hwang \\ Department of Engineering Sciences, Physics and Mathematics, \\ Karlstad University, S-651 88 Karlstad, Sweden \\ and \\ Theory division, C.E.R.N. \\ CH-1211 Geneva 23, Switzerland
}

\begin{abstract}
If $G$ is a simple non-compact Lie Group, with $K$ its maximal compact subgroup, such that $K$ contains a one-dimensional center $C$, then the coset space $G / K$ is an Hermitian symmetric non-compact space. $S L(2, \mathbb{R}) / U(1)$ is the simplest example of such a space. It is only when $G / K$ is an Hermitian symmetric space that there exists unitary discrete representations of $G$. We will here study string theories defined as $G / K^{\prime}, K^{\prime}=K / C$, WZNW models. We will establish unitarity for such string theories for certain discrete representations. This proof generalizes earlier results on $S L(2, \mathbb{R})$, which is the simplest example of this class of theories. We will also prove unitarity of $G / K$ conformal field theories generalizing results for $S L(2, \mathbb{R}) / U(1)$. We will show that the physical space of states lie in a subspace of the $G / K$ state space.
\end{abstract}

Accepted for publication in Physics Letters B

\footnotetext{
1email: stephen.hwang@hks.se and stephen.hwang@cern.ch
} 
Although string theories have been extensively studied for several decades they still provide many surprises. String dualities are one of the more recent examples. In this paper we will study a class of string theories which provides one of the counterexamples to the successes of string theories. For string theories propagating on non-compact curved backgrounds little is known although some amount of effort has been put into investigations. The simplest example is the so-called $S L(2, \mathbb{R}$ ) (or $S U(1,1)$ or $S O(2,1)$ ) string theory. This theory is a $S L(2, \mathbb{R})$ WZNW model with conformal anomaly $c=26$. For this case it was shown [1] that a selection of discrete representations similar to that of integrable representations for compact affine Lie algebras, gave unitarity in the physical subspace. In [2] a proposal of a modular invariant partition function was given. This proposal was further discussed in [3]. Still its status is an open question as it contains divergencies that seem to resist regularization. The question of consistency of the selection of representations in an interacting theory is furthermore an unresolved issue. A discussion has been given in [3]. A proposal for another solution was given in [4].

Recent developments have renewed the interest in string theories on noncompact curved manifolds. One of these is due to Maldacena [5], who suggested that string theories on a $d+1$ dimensional Anti de Sitter $(A d S)$ space times some compact manifold is related to the large $\mathrm{N}$ limit of conformally invariant $\mathrm{SU}(\mathrm{N})$ gauge theories. Another development is due to Strominger [6], who made a simple and elegant derivation of the Beckenstein-Hawking entropy formula for three dimensional black holes, whose near-horizon symmetry is $A d S_{3}$. This derivation uses the fact [7] that any consistent quantum gravity theory on $A d S_{3}$ is a two-dimensional conformal field theory (CFT). As $A d S_{3}$ is the group manifold of $S L(2, \mathbb{R})$ it is suggestive that the relevant CFT giving the correct number of states needed for the derivation in $[6]$ is related to the $S L(2, \mathbb{R})$ CFT. A derivation using this CFT was given by Carlip [8]. A proposal for the CFT that describes $A d S_{3}$ has been given in [9] and involves a $S L(2, \mathbb{R}) / U(1) \mathrm{CFT}$.

In this work we will not attempt to address the unresolved issues of the $S L(2, R)$ string theory and related CFT's. Instead, we will focus on generalizations of these theories, with the hope that once the $S L(2, \mathbb{R})$ model is fully understood the implications on the generalized theories considered here will be straightforward. There are two natural generalizations to the $S L(2, \mathbb{R})$ theory with one "time coordinate". One general class of theories of which 
$S L(2, \mathbb{R})$ is the simplest example of is AdS spaces of arbitrary dimension with one "time-like" direction [10], [11]. Such theories are of course highly interesting due to Maldacena's results. They can be realized as the coset spaces $S O(d-1,2) / S O(d-1,1)$.

Another class of generalizations with one "time coordinate" is the following. Let $G$ be a non-compact Lie group and $K$ its maximal compact subgroup. Require that $K$ contains a one-dimensional center $C$. The space $G / K^{\prime}, K^{\prime}=$ $K / C$, has one "time-like" (compact) direction and the simplest example is $S L(2, \mathbb{R})$. The spaces $G / K$ are in these cases Hermitian symmetric spaces. A classification of these spaces may be found in [12]. There are four large classes: $S U(p, q) / S(U(p) \times U(q)), S O(p, 2) / S O(p) \times S O(2), S O^{*}(2 n) / U(n)$ and $S p(n, \mathbb{R}) / U(n)$. In addition there is one where $G$ is a certain real form of $E_{6}^{\mathbb{C}}$ and another where it is a real form of $E_{7}^{\mathbb{C}}$. The dimension of the spaces are $d=2 p q, 2 p, n(n-1), n(n+1), 32$ and 54 , respectively. Interestingly enough, it is precisely when $G / K$, where $K$ refers to the maximal compact subgroup of $G$, is a Hermitian symmetric space that $G$ admits unitary highest weight modules [13, 114] (this is easily understood from the fact that only in these cases can we choose the Cartan subgroup to lie completely in $K$ ). These unitary representations have been completely classified in [15], [16]. For any other non-compact group the unitary representations are necessarily continous. Comparatively little is known about them in the general case. This is one of the reasons that we have chosen to study the second class of string theories. Another reason is the possibility to generalize the $S L(2, \mathbb{R}) / U(1)$ construction, which is interesting due to its interpretation as a two-dimensional black hole [17. The two above classes, are the only ones for $G$ simple which have one "time-coordinate" (see [18], where also a number of possibilities for $G$ being a product of simple factors are given).

Our main result, given in the Theorem, will be to establish unitarity for string theories defined as $G / K^{\prime}$ WZNW models times some arbitrary unitary CFT for certain highest weight representations of $\mathfrak{g}$, the affine Lie algebra corresponding to $G$. These representations have highest weights $\lambda$ that are antidominant. In the course of proving unitarity we will generalize the result of Dixon, Lykken and Peskin [19] on the unitarity of $S L(2, \mathbb{R}) / U(1)$ for discrete representations to $G / K$ for arbitrary Hermitian symmetric pairs $(G, K) \rrbracket$. This

\footnotetext{
${ }^{2}$ In the string context $G / K^{\prime}$ is not completely arbitrary as the total conformal anomaly
} 
result is given in the Proposition. We will in fact prove that the genuine physical state space is a subspace of the space defined by $G / K$.

The techniques used to prove the Theorem are not the same as in the original proof [1]. The reason is that the latter proof relied on the use of a continous basis, which is both unnatural and we do not know properties of its generalization to the present case. Instead more relevant references to the present case are [20] and [21] (relying to some extent on techniques in 22]).

We consider $G$ to be a simple finite dimensional Lie group over $\mathbb{R}$ of noncompact type and $(G, K)$ a Hermitian symmetric pair. We denote by $\overline{\mathfrak{g}}_{0}$ and $\overline{\mathfrak{k}}_{0}$ the Lie algebra of $G$ and $K$, respectively and let $\overline{\mathfrak{g}}_{0}=\overline{\mathfrak{k}}_{0}+\overline{\mathfrak{p}}_{0}$ be a Cartan decomposition i.e. a decomposition into compact and non-compact pieces. Let $\overline{\mathfrak{h}}_{0} \subseteq \overline{\mathfrak{k}}_{0}$ be a Cartan subalgebra (CSA). Then $\overline{\mathfrak{h}}_{0}$ is also a CSA of $\overline{\mathfrak{g}}_{0}$. Furthermore, $\overline{\mathfrak{k}}_{0}=\overline{\mathfrak{k}}_{0}^{\prime}+\overline{\mathfrak{c}}_{0}$, where $\overline{\mathfrak{k}}_{0}^{\prime}$ is a semi-simple algebra and $\overline{\mathfrak{c}}_{0} \in \overline{\mathfrak{h}}_{0}$ is a one-dimensional center of $\overline{\mathfrak{k}}_{0}$. Let $\overline{\mathfrak{g}}, \overline{\mathfrak{k}}$ be the complexifications of $\overline{\mathfrak{g}}_{0}, \overline{\mathfrak{k}}_{0}$ etc. We denote by $\bar{\Delta}$ the set of roots of $\overline{\mathfrak{g}}$ relative to $\overline{\mathfrak{h}}$ and $\bar{\Delta}=\bar{\Delta}_{\mathfrak{k}} \cup \bar{\Delta}_{\mathfrak{p}}$ is the decomposition of $\bar{\Delta}$ into compact roots $\bar{\Delta}_{\mathfrak{k}}$ and non-compact roots $\bar{\Delta}_{\mathfrak{p}}$. We fix an ordering of $\bar{\Delta}$ and $\bar{\Delta}^{+}$denotes the set of positive roots and $\overline{\mathfrak{g}}=\overline{\mathfrak{n}}^{-}+\overline{\mathfrak{h}}+\overline{\mathfrak{n}}^{+}$is a triangular decomposition w.r.t. this ordering. Define $\bar{\Delta}_{\mathfrak{p}}^{+} \subset \bar{\Delta}^{+}$and $\bar{\Delta}_{\mathfrak{k}}^{+} \subset \bar{\Delta}^{+}$ to be the corresponding positive roots for $\overline{\mathfrak{p}}$ and $\overline{\mathfrak{k}}$ and $\bar{\rho}=\sum_{\bar{\alpha} \in \bar{\Delta}^{+}} \bar{\alpha}$. Let $\bar{\Delta}^{s}=\left\{\bar{\alpha}_{1}, \ldots, \bar{\alpha}_{r}\right\} \subset \bar{\Delta}^{+}$be the set of simple roots. It is always possible to choose an ordering of $\bar{\Delta}$ such that $\bar{\alpha}_{i}>\bar{\alpha}_{j}$ for all $\bar{\alpha}_{i} \in \bar{\Delta}_{\mathfrak{p}}$ and all $\bar{\alpha}_{j} \in \bar{\Delta}_{\mathfrak{k}}$. This is accomplished by taking the first entry of $\bar{\alpha}$ to be the eigenvalue with respect to $h \in \overline{\mathfrak{c}}$. Since $\overline{\mathfrak{c}}$ is in the center of $\overline{\mathfrak{k}}$, roots of $\overline{\mathfrak{k}}$ will have a zero as first entry. We have $\bar{\alpha}_{i}+\bar{\alpha}_{j} \notin \bar{\Delta}$ if $\bar{\alpha}_{i}, \bar{\alpha}_{j} \in \bar{\Delta}_{\mathfrak{p}}^{+}$. Furthermore, the highest root $\bar{\theta}$ of $\bar{\Delta}$ is in $\bar{\Delta}_{\mathfrak{p}}$ and $\bar{\Delta}^{s} \cap \bar{\Delta}_{\mathfrak{p}}^{+}$contains a single root. We define Hermite conjugation $x^{\dagger}$ of $x \in \mathfrak{g}$ such that elements in $\overline{\mathfrak{g}}_{0}$ are Hermitian. Then $h^{\dagger}=h,\left(e^{\alpha}\right)^{\dagger}= \pm e^{-\alpha}$, where the plus sign occurs when $\bar{\alpha} \in \bar{\Delta}_{\mathfrak{k}}$.

We extend the finite dimensional algebras $\overline{\mathfrak{g}}_{0}$ and $\overline{\mathfrak{g}}$ to the corresponding affine Lie algebras $\mathfrak{g}_{0}$ and $\mathfrak{g}$. The Cartan decomposition of $\overline{\mathfrak{g}}$ extends naturally to a decomposition $\mathfrak{g}=\mathfrak{k}+\mathfrak{p}$, where generators of $\mathfrak{g}$ satisfy $h^{\dagger}=h$ for $h \in \mathfrak{h}$, $\left(e^{\alpha}\right)^{\dagger}= \pm e^{-\alpha}$, where the plus sign occurs when $\alpha \in \Delta_{\mathfrak{k}}$. $\mathfrak{k}$ admits a further decomposition $\mathfrak{k}=\mathfrak{k}^{\prime}+\mathfrak{c}$ induced by the corresponding decomposition of the satisfies $c=26$. This requires that $\operatorname{dim}\left(G / K^{\prime}\right) \leq 26$. For the unitarity of the coset $G / K$ there will be no such restriction. 
finite dimensional algebra. We denote by $\Delta$ the roots of $\mathfrak{g}$ etc. for $\Delta_{\mathfrak{k}}, \Delta_{\mathfrak{c}}$. $\Delta^{s}$ and $\Delta^{+}$denote simple and positive roots, respectively. Denote by $\alpha_{\mathfrak{c}}$ the roots $\alpha \in \Delta_{\mathfrak{c}}^{+}$.

The string theory that we will consider here has a state space which is the irreducible space $\mathcal{L}^{\left(\mathfrak{g}, \mathfrak{k}^{\prime}\right)}(\lambda) \times \mathcal{M}^{\prime}$ of $\mathcal{M}^{\left(\mathfrak{g}, \mathfrak{k}^{\prime}\right)}(\lambda) \times \mathcal{M}^{\prime}$, where $\mathcal{M}^{\prime}$ is the state space of an arbitrary unitary conformal field theory and $\mathcal{M}^{\left(\mathfrak{g}, \mathfrak{k}^{\prime}\right)}$ is formed by taking the highest weight $\mathfrak{g}$ Verma module $\mathcal{M}^{(\mathfrak{g})}(\lambda)$ of highest weight $\lambda$ and requiring that the vectors $|v\rangle \in \mathcal{M}^{\left(\mathfrak{g}, \mathfrak{k}^{\prime}\right)}(\lambda)$ are highest weight vectors with respect to $\mathfrak{k}^{\prime}$ i.e. $e^{\alpha}|v\rangle=0$ for all roots $\alpha \in \Delta_{\mathfrak{k}^{\prime}}^{+}$. In the simplest case, $\mathfrak{g}=\hat{s l}(2, \mathbb{R}), \mathcal{M}(\lambda)=$ $\mathcal{M}^{\left(\mathfrak{g}, \mathfrak{k}^{\prime}\right)}(\lambda)$. The Virasoro generators $L_{n}$ relevant for our construction have the form $L_{n}=L_{n}^{\left(\mathfrak{g}, \mathfrak{k}^{\prime}\right)}+L_{n}^{\prime}$. Here $L_{n}^{\left(\mathfrak{g}, \mathfrak{k}^{\prime}\right)}=L_{n}^{(\mathfrak{g})}-L_{n}^{\left(\mathfrak{k}^{\prime}\right)}$ is the difference between the Virasoro generators formed from generators of $\mathfrak{g}$ and $\mathfrak{k}^{\prime}$, respectively, in the usual way. We denote by $L_{n}^{(\mathfrak{g}, \mathfrak{k})}=L_{n}^{\left(\mathfrak{g}, \mathfrak{k}^{\prime}\right)}-L_{n}^{(\mathfrak{c})}$, where $L_{n}^{(\mathfrak{c})}$ is the time-like $\hat{u}(1)$ Virasoro generator, where time-like refers to the sign in the commutation relations in the free field limit $k \rightarrow-\infty$. $k$ is here the central element of $\mathfrak{g}$. The central charge of the Virasoro algebra generated by $L_{n}$ is assumed to be 26 . It is of the form $c=c_{\mathfrak{g}}-c_{\mathfrak{k}^{\prime}}+c^{\prime}$, where $c_{\mathfrak{g}}=\frac{k d_{\overline{\mathfrak{g}}}}{k+c_{\overline{\mathfrak{g}}}}, c_{\mathfrak{k}^{\prime}}=\frac{k d_{\overline{\mathfrak{k}^{\prime}}}}{k+c_{\overline{\mathfrak{k}^{\prime}}}}$ for $\mathfrak{k}^{\prime}$ simple and $c^{\prime}$ is the central charge of the CFT defined on $\mathcal{M}^{\prime}$. Here $d_{\overline{\mathfrak{g}}}$ is the dimension of $\overline{\mathfrak{g}}$ and $c_{\overline{\mathfrak{g}}}$ is the second Casimir of the adjoint representation of $\overline{\mathfrak{g}}$ etc. for $\mathfrak{k}^{\prime}$. Reparametrization invariance of string theories requires that physical states $|\phi\rangle$ should satisfy the Virasoro conditions

$$
\begin{aligned}
& L_{n}|\phi\rangle=0, \quad n>0, \\
& \left(L_{0}-1\right)|\phi\rangle=0 .
\end{aligned}
$$

The result we will establish is the following.

Theorem. The solution of the eqs. (11) and (2) in $\mathcal{L}^{\left(\mathfrak{g}, \mathfrak{k}^{\prime}\right)}(\lambda) \times \mathcal{M}^{\prime}$ with $\lambda$ being antidominant are of the form

$$
|\phi\rangle=|t\rangle+|n\rangle
$$

where $|t\rangle$ satisfies eqs. (11) and (2) and is a highest weight vector w.r.t. $\mathfrak{k}$ and $|n\rangle$ is a physical null state, i.e. a state which decouples from all physical states. Furthermore, the states $|\phi\rangle$ form a unitary state space. 
Here a weight $\lambda$ is defined to be antidominant if $(2 \lambda+\rho) \cdot \alpha \leq 0$ for all simple roots of $\mathfrak{g}$, where $\rho$ is defined by $\rho \cdot \alpha=\alpha^{2}$. Antidominant weights require $k+c_{\overline{\mathfrak{g}}}<0$. Note that although $|t\rangle \in \mathcal{L}^{(\mathfrak{g}, \mathfrak{k})}(\lambda) \times \mathcal{M}^{\prime}$, the precise form of $\mathcal{M}^{\prime}$ will be unimportant and we will henceforth surpress its presence. The proof of the theorem will be divided into several steps. Our first step is to establish the following.

Lemma 1. A basis of states of $\mathcal{L}^{\left(\mathfrak{g}, \mathfrak{k}^{\prime}\right)}$ with $\lambda$ antidominant is

$$
|\psi\rangle=L_{-n_{1}} \ldots L_{-n_{k}} e^{-\alpha_{1}} \ldots e^{-\alpha_{k^{\prime}}}|l, m\rangle,
$$

where $n_{1}, \ldots n_{k}>0, \alpha_{1}, \ldots, \alpha_{k^{\prime}} \in \Delta_{\mathfrak{c}}^{+}, n_{i} \leq n_{j}$ and $\alpha_{i} \leq \alpha_{j}$ for $i<j$, and $|l, m\rangle$ is highest weight w.r.t. $\operatorname{Vir}(L)$, the Virasoro algebra generated by $L_{n}$, and $\mathfrak{c}$ with weights $l$ and $m$, respectively.

Proof. Let $\mathcal{V}$ denote the largest irreducible subspace of $\mathcal{L}^{\left(\mathfrak{g}, \mathfrak{k}^{\prime}\right)}$ that has a basis of the following form

$$
|\psi\rangle=L_{-n_{1}}^{(\mathfrak{g}, \mathfrak{k})} \ldots L_{-n_{k}}^{(\mathfrak{g}, \mathfrak{k})} e^{-\alpha_{1}} \ldots e^{-\alpha_{k^{\prime}}}\left|l^{(\mathfrak{g}, \mathfrak{k})}\right\rangle \otimes|m\rangle,
$$

where $L_{n}^{(\mathfrak{g}, \mathfrak{k})}=L_{n}-L_{n}^{(\mathfrak{c})}$ and $\left|l^{(\mathfrak{g}, \mathfrak{k})}\right\rangle \otimes|m\rangle$ is a highest weight state w.r.t. $\operatorname{Vir}\left(L^{(\mathfrak{g}, \mathfrak{k})}\right)+\mathfrak{c}$ with weights $l^{(\mathfrak{g}, \mathfrak{k})}, m$. This space certainly exists. As $\mathcal{V}$ is irreducible the matrix $H\left(l^{(\mathfrak{g}, \mathfrak{k})}, m\right)$ of inner products restricted to vectors in $\mathcal{V}$ has a non-vanishing determinant. Hence, we may form the orthogonal complement $\mathcal{V}^{\prime}$ to $\mathcal{V}$ with respect to the inner product. Assume that $\mathcal{V}^{\prime}$ is non-trivial and let $|v\rangle \in \mathcal{V}^{\prime}$ be a vector that has the smallest $L_{0}$ eigenvalue in $\mathcal{V}^{\prime}$. Such a vector certainly exists if $\mathcal{V}^{\prime}$ is non-trivial. Consider $\left|v_{1}\right\rangle=x|v\rangle$, where $x=L_{n}^{(\mathfrak{g}, \mathfrak{k})}$ for some value $n>0$ or $x=e^{\alpha_{\mathfrak{c}}}$. Then $\left|v_{1}\right\rangle \in \mathcal{V}$. If $\left|v_{1}\right\rangle$ is zero for all $n$ and all $\alpha_{\mathfrak{c}}$, then $|v\rangle \in \mathcal{V}$, and $\mathcal{V}^{\prime}$ is trivial. Denote by $\left|v_{2}\right\rangle$ a vector in $\mathcal{V}$ such that $\left\langle v_{2} \mid v_{1}\right\rangle \neq 0$. $\left|v_{2}\right\rangle$ exists as $\mathcal{V}$ is irreducible. We have $0 \neq\left\langle(x v) \mid v_{2}\right\rangle=\left\langle v \mid x^{\dagger} v_{2}\right\rangle$. We will now prove that either $x^{\dagger}\left|v_{2}\right\rangle \in \mathcal{V}$ or it is zero. In both cases the r.h.s. is zero (in the former case from the orthogonality between $\mathcal{V}$ and $\mathcal{V}^{\prime}$ ). Thus, we will have a contradiction and $\mathcal{V}^{\prime}$ is trivial.

Consider $|w\rangle=x^{\dagger}\left|v_{2}\right\rangle$ for any $\left|v_{2}\right\rangle \in \mathcal{V} .|w\rangle \in \mathcal{V}$ except in the cases where $|w\rangle$ is outside the irreducible submodule. As the $\mathfrak{c}$ Verma module is irreducible, this can only happen if there are singular vectors in the $\operatorname{Vir}\left(L^{(\mathfrak{g}, \mathfrak{k})}\right)$ module. The singular vectors may be determined by reading off the zeros of the Kac 
determinant corresponding to these modules. We have $c=25$ and for this case the Kac determinant has zeros for $l^{(\mathfrak{g}, \mathfrak{k})}=1-\frac{(p+q)^{2}}{4}$, where $p, q>0$ and integers. For antidominant weights we have $l^{(\mathfrak{g}, \mathfrak{k})} \geq 0$ with equality only for $k \rightarrow-\infty$. This may be shown by carefully checking the explicit form of $L_{0}^{(\mathfrak{g}, \mathfrak{k})}$ (the first property, $l^{(\mathfrak{g}, \mathfrak{k})} \geq 0$, follows, however, immediately from the Proposition below). For finite values of $k$ the Virasoro Verma module is irreducible and, hence, $x^{\dagger}\left|v_{2}\right\rangle \in \mathcal{V}$. For $k \rightarrow-\infty$ we have a null-vector for $p=q=1$ and $l^{(\mathfrak{g}, \mathfrak{k})}=0$. This null-vector is $|n v\rangle=L_{-1}^{(\mathfrak{g}, \mathfrak{k})}\left|l^{(\mathfrak{g}, \mathfrak{k})}=0\right\rangle$. The $\mathfrak{g}$ module becomes a Fock module as $k \rightarrow-\infty$ and in this case $|n v\rangle$ is identically zero. Thus, we have either $x^{\dagger}\left|v_{2}\right\rangle \in \mathcal{V}$ or it is zero. We have proved our claim. The lemma follows now by replacing $L_{n}^{(\mathfrak{g}, \mathfrak{k})}$ by $L_{n}$ using $L_{n}^{(\mathfrak{g}, \mathfrak{k})}=L_{n}-L_{n}^{(\mathfrak{c})}$ and commuting all the $L_{n}$ 's to the left.

We may by (4) uniquely decompose $|\psi\rangle$ as

$$
|\psi\rangle=|\phi\rangle+|s\rangle
$$

Here $|\phi\rangle \in \mathcal{P}$ is a state of the form $|\phi\rangle=\prod e^{-\alpha_{\mathfrak{c}}}|l\rangle \otimes|m\rangle$ and $|s\rangle \in \mathcal{S}$ is a so-called spurious state i.e. of the form $|s\rangle=L_{-n}|\chi\rangle$ for some $n>0$ and $|\chi\rangle$. The next lemma is due to Goddard and Thorn [22].

Lemma 2. For $|\psi\rangle$ of the form eq. (6), the equations

$$
L_{n}|\psi\rangle=\left(L_{0}-1\right)|\psi\rangle=0, \quad n>0
$$

are equivalent for $c=26$ to

$$
\begin{aligned}
L_{n}|\phi\rangle & =\left(L_{0}-1\right)|\phi\rangle=0, & & n>0, \\
L_{n}|s\rangle & =\left(L_{0}-1\right)|s\rangle=0 . & & n>0,
\end{aligned}
$$

Lemma 3. A basis of $\mathcal{L}^{(\mathfrak{g})}(\lambda)$, the irreducible module of $\mathcal{M}^{(\mathfrak{g})}(\lambda)$, is for antidominant weights $\lambda$

$$
|v\rangle=e^{-\alpha_{1}} \ldots e^{-\alpha_{n}}\left|\lambda_{\mathfrak{k}}\right\rangle, \quad \alpha_{1}, \ldots \alpha_{n} \in \Delta_{\mathfrak{k}}^{s} \cup \Delta_{\mathfrak{c}}^{+}
$$

Here $\left|\lambda_{\mathfrak{k}}\right\rangle$ is a highest weight state w.r.t. $\mathfrak{k}$ with weight $\lambda_{\mathfrak{k}}$.

Proof. The Kac-Kazhdan determinant formula for states with weight $\lambda-\eta$ in 
$\mathcal{M}^{(\mathfrak{g})}(\lambda)$ reads 23

$$
\operatorname{det} H_{\eta}=C_{1} \prod_{\alpha \in \Delta^{+}} \prod_{n=1}^{\infty}[(2 \lambda+\rho, \alpha)-n(\alpha, \alpha)]^{P(\eta-n \alpha)},
$$

where $C_{1}$ is a non-zero constant. From this formula it is evident that for $\lambda$ being antidominant $\mathcal{M}^{(\mathfrak{g})}(\lambda)$ is irreducible i.e. $\mathcal{M}^{(\mathfrak{g})}(\lambda)$ and $\mathcal{L}^{(\mathfrak{g})}(\lambda)$ are isomorphic. Denote by $\mathcal{V}$ the largest irreducible space that has $(9)$ as a basis. As $\mathcal{M}^{(\mathfrak{g})}(\lambda)$ is irreducible it follows that if $|v\rangle \in \mathcal{V}$ then $x^{\dagger}|v\rangle \in \mathcal{V}$ for any $x \in \mathfrak{k}$. By exactly the same argument that lead us to conclude that (5) was a basis, we may conclude that the orthogonal complement $\mathcal{V}^{\prime}$ to $\mathcal{V}$ is trivial and hence (9) is a basis.

Proposition. The space $\mathcal{L}^{(\mathfrak{g}, \mathfrak{k})}(\lambda)$ is unitary for $\lambda$ being antidominant.

Proof. We have by Lemma 3 that the Kac-Kazhdan determinant for $\mathcal{M}^{(\mathfrak{g})}(\lambda)$ can be written in the form

$$
\operatorname{det} H=\operatorname{det} H^{(\mathfrak{k})} \operatorname{det} H^{(\mathfrak{g}, \mathfrak{k})}
$$

Here $H^{(\mathfrak{g}, \mathfrak{k})}$ arises from states in $\mathcal{L}^{(\mathfrak{g}, \mathfrak{k})}$ and $H^{(\mathfrak{k})}$ from all other states, which by Lemma 3 are non-highest weight states in $\mathfrak{k}$ modules. As $\operatorname{det} H$ is non-zero it follows that $\operatorname{det} H^{(\mathfrak{g}, \mathfrak{k})}$ is non-zero. This implies that the signature of $H^{(\mathfrak{g}, \mathfrak{k})}$ remains unchanged for any antidominant value of $\lambda$. We consider the limit $k \rightarrow-\infty$, which is a possible limit for antidominant weights. This limit is just the free field limit in which $\mathfrak{g}$ reduces to a direct sum of Fock algebras. Then a basis of $\mathcal{L}^{(\mathfrak{g}, \mathfrak{k})}$ are states of the form

$$
\left|v^{(\mathfrak{g}, \mathfrak{k})}\right\rangle=e^{-\alpha_{1}} \ldots e^{\prime-\alpha_{n}}|\lambda\rangle, \quad \alpha_{i} \leq \alpha_{j} \text { for } i \leq j, \quad \alpha_{1}, \ldots, \alpha_{n} \in \Delta_{\mathfrak{p}}^{+}
$$

where $e^{\prime-\alpha_{i}}=\frac{1}{\sqrt{-k}} e^{-\alpha_{i}}$ and $|\lambda\rangle$ is the highest weight state generating $\mathcal{M}^{(\mathfrak{g})}(\lambda)$. As $e^{\prime-\alpha_{i}}$ act as free field modes with a positive signature, it is evident that $H^{(\mathfrak{g}, \mathfrak{k})}$ has a positive definite signature in this limit and hence for all antidominant weights.

As remarked in the proof of Lemma 1, an immediate consequence of the Proposition is that $L_{0}^{(\mathfrak{g}, \mathfrak{k})}$ has non-negative eigenvalues in $\mathcal{L}^{(\mathfrak{g}, \mathfrak{k})}$. The Proposition gives a generalization of the unitarity theorem given in [19] for the case 
$S L(2, \mathbb{R}) / U(1)$. Notice that the Proposition is true even for $c \neq 26$.

Proof of Theorem. By Lemma 1 and Lemma 2 we have that physical states should satisfy eq. (\$). As any state in $\mathcal{S}$ decouples from all physical states it is sufficient to study states in $\mathcal{P}$ satisfying eq. (8). By Lemma 1 we can choose a basis of $\mathcal{P}$ consisting of states $|t\rangle \in \mathcal{T}$, which are highest weight states w.r.t. the algebra $\operatorname{Vir}(L)+\mathfrak{c}$, and $|u\rangle \in \mathcal{U}$ which are created from $|t\rangle$ by acting with $e^{-\alpha_{\mathfrak{c}}}$. Decomposing $|\phi\rangle \in \mathcal{P}$ as $|\phi\rangle=|t\rangle+|u\rangle$ it follows that $L_{n}|\phi\rangle=0$ reduces to the equation $L_{n}|u\rangle=0$, which in turn reduces to $L_{n}^{(\mathfrak{c})}|u\rangle=0$. The $\mathfrak{c}$ module is a Fock module and it is well-known that there are no non-trivial on-shell states, except for $m=0$ ․ For $m=0$ we have a state $\left|u_{1}\right\rangle=e^{-\alpha_{\mathfrak{c}}}|l, m=0\rangle$, where $|l, m=0\rangle \in \mathcal{T}$ and $\left[L_{0}, e^{-\alpha_{\mathfrak{c}}}\right]=e^{-\alpha_{\mathfrak{c}}}$, which satisfies $L_{n}\left|u_{1}\right\rangle=0, n>0$. The on-shell condition requires $l=0$ so that $l^{(\mathfrak{g}, \mathfrak{k})}=0$, which in turn requires $k \rightarrow-\infty$ (cf. the proof of Lemma 1). In this free field limit it is easy to see and well-known that there are no on-shell states in $\mathcal{U}$ that have $m=0$. Thus in the space $\mathcal{P}$, only states in $\mathcal{T}$ are physical. This is the first claim of the theorem. The second claim is that this subspace is unitary and it follows from the Proposition.

Note that $\mathcal{T}$ is a subspace of $\mathcal{L}^{(\mathfrak{g}, \mathfrak{k})}$ and, hence, the genuine physical states are in $\mathcal{L}^{(\mathfrak{g}, \mathfrak{k})}$.

One relevant question is whether it is necessary to assume antidominant weights for unitarity. If one considers the free field limit $k \rightarrow-\infty$, then it is easily seen that a basis of $\mathcal{L}^{(\mathfrak{g}, \mathfrak{k})}$ is given by states of the form $e^{\prime-\alpha_{1}} \ldots e^{\prime-\alpha_{n}}|\lambda\rangle$, $\alpha_{i} \in \Delta_{\mathfrak{p}}^{+}$. Unitarity of this state space then requires $\lambda \cdot \alpha \leq 0$ for $\alpha \in \Delta_{\mathfrak{p}}^{+}$. Our conjecture is, therefore, that it is sufficient to require $(2 \lambda+\rho) \cdot \alpha \leq 0, \alpha \in \Delta_{\mathfrak{p}}^{+}$ to ensure unitarity.

Acknowledgement: I would like to thank Steve Carlip and Genkai Zhang for helpful discussions.

\footnotetext{
${ }^{3}$ This special case was overlooked in [21]
} 


\section{References}

[1] S. Hwang, Nucl. Phys. B351 (1991) 425

[2] M. Henningson, S. Hwang, P. Roberts and B. Sundborg, Phys. Lett. B267 (1991) 350

[3] S. Hwang and P. Roberts, Proceedings of the 16'th Johns Hopkins' workshop, ed. by L. Brink and R. Marnelius, World Scientific 1993 hepth/9211075)

[4] I. Bars, Phys. Rev. D53 (1996) 3308

[5] J. Maldacena, hep-th/9711200

[6] A. Strominger, hep-th/9712251

[7] J.D. Brown and M. Henneaux, Comm. Math. Phys. 104 (1986) 207

[8] S. Carlip, Phys. Rev. D51 (1995) 632

[9] K. Behrndt, I. Brunner and I. Gaida, hep-th/9804159

[10] E.S. Fradkin and V.Ya. Linetsky, Phys. Lett. B261 (1991) 26

[11] I. Bars, D. Nemenschansky, Nucl. Phys. B348 (1991) 89

[12] S. Helgason, Differential geometry, Lie groups, and symmetric spaces, Academic Press, 1978

[13] Harish-Chandra, Amer. J. Math. 77 (1955), 743

[14] Harish-Chandra, Amer. J. Math. 78 (1956), 1

[15] H.P. Jakobsen, J. Funct. Anal. 52 (1983) 385-412

[16] T. Enright, R. Howe and N. Wallach, Representation theory of reductive groups, Proceedings of the university of Utah Conference 1982, Birkhäuser, Boston 1983, p 97-143

[17] E. Witten, Phys. Rev. D44 (1991) 314

[18] P. Ginsparg and F. Quevedo, Nucl. Phys. B385 (1992) 527 
[19] L.J. Dixon, J. Lykken and M.E. Peskin, Nucl. Phys. B325 (1989) 329

[20] C. Bachas and S. Hwang, Phys. Lett. B247 (1990) 265

[21] S. Hwang, Phys. Lett. B276 (1992) 451

[22] P. Goddard and C. Thorn, Phys. Lett. B40 (1972) 235

[23] V.G. Kac and D.A. Kazhdan, Adv. in Math. 34 (1979) 97 